KYUNGPOOK Math. J. 54(2014), 1-9

http://dx.doi.org/10.5666/KMJ.2014.54.1.1

\title{
Hilbert-Type Integral Inequalities in the Whole Plane with the Non-homogeneous Kernel
}

\section{DONGMEI XiN}

Department of Mathematics, Guangdong University Education, Guangzhou, Guangdong 510303, China

e-mail : xdm77108@gdei.edu.cn

Abstract. We build new Hilbert-type integral inequalities in the whole plane with the non-homogeneous kernel involving some parameters and the best constant factors. We also consider their reverse.

\section{Introduction}

If $f(x), g(x) \geq 0$ satisfying $0<\int_{0}^{\infty} f^{2}(x) d x<\infty$ and $0<\int_{0}^{\infty} g^{2}(x) d x<\infty$, then we have a space(see [1])

$$
\int_{0}^{\infty} \int_{0}^{\infty} \frac{f(x) g(y)}{x+y} d x d y<\pi\left(\int_{0}^{\infty} f^{2}(x) d x \int_{0}^{\infty} g^{2}(x) d x\right)^{\frac{1}{2}},
$$

where the constant factor $\pi$ is the best possible. Inequality (1.1) is well known as Hilbert's integral inequality, which is important in analysis and applications ${ }^{[1-2]}$. By Inequality (1.1), we can get a Hilbert-type integral inequality with a nonhomogeneous kernel as follows ${ }^{[3]}$ :

$$
\int_{-\infty}^{\infty} \int_{-\infty}^{\infty} \frac{f(x) g(y)}{|1+x y|^{\lambda}} d x d y<k_{\lambda}\left(\int_{-\infty}^{\infty} f^{p}(x) d x\right)^{\frac{1}{p}}\left(\int_{-\infty}^{\infty} g^{q}(x) d x\right)^{\frac{1}{q}}
$$

Some inequalities with the non-homogenous kernels have been studied(see[4-9]).

By applying the method for (1.2) and using the way of real analysis, the main objective of this paper is to give new Hilbert-type integral inequalities in the whole plane with the non-homogeneous kernel involving some parameters and best constant factors. Their reverse forms are also considered. As applications, we also obtain the equivalent forms and some particular cases.

Received April 25, 2011; accepted September 23, 2011.

2010 Mathematics Subject Classification: 26D15.

Key words and phrases: Hilbert-type integral inequality, Whole Plane, Non-Homogeneous, weight function. 


\section{Some Lemmas}

Lemma 2.1. If $0<\alpha_{1}<\alpha_{2}<\pi$, define the weight function $\omega(x)(x \in(-\infty, \infty))$ as follow:

$$
\omega(x):=\int_{-\infty}^{\infty} \min _{i \in\{1,2\}}\left\{\frac{1}{1+2 x y \cos \alpha_{i}+(x y)^{2}}\right\}|x| d y,
$$

Then we have $\omega(x) \neq 0$, where

$$
k:=\frac{\alpha_{1}}{\sin \alpha_{1}}+\frac{\pi-\alpha_{2}}{\sin \alpha_{2}} .
$$

Proof. For $x \in(-\infty, 0)$, setting $u=x y, u=-x y$ respectively in the following first and second integrals, we have

$$
\begin{aligned}
\omega(x) & =\int_{-\infty}^{0} \frac{-x}{1+2 x y \cos \alpha_{1}+(x y)^{2}} d y \\
& +\int_{0}^{\infty} \frac{-x}{1+2 x y \cos \alpha_{2}+(x y)^{2}} d y \\
& =\int_{0}^{\infty} \frac{1}{u^{2}+2 u \cos \alpha_{1}+1} d u+\int_{0}^{\infty} \frac{1}{u^{2}-2 u \cos \alpha_{2}+1} d u \\
& =\frac{\alpha_{1}}{\sin \alpha_{1}}+\frac{\pi-\alpha_{2}}{\sin \alpha_{2}} .
\end{aligned}
$$

For $x \in(0, \infty)$, setting $u=-x y, u=x y$ respectively in the following first and second integrals, we have

$$
\begin{aligned}
\omega(x) & =\int_{-\infty}^{0} \frac{x}{1+2 x y \cos \alpha_{2}+(x y)^{2}} d y \\
& +\int_{0}^{\infty} \frac{x}{1+2 x y \cos \alpha_{1}+(x y)^{2}} d y \\
& =\int_{0}^{\infty} \frac{1}{u^{2}-2 u \cos \alpha_{2}+1} d u+\int_{0}^{\infty} \frac{1}{u^{2}+2 u \cos \alpha_{1}+1} d u=k .
\end{aligned}
$$

The lemma is proved.

Note. (1) It is obvious that $\omega(0)=0$;

(2) If $\alpha_{1}=\alpha_{2}=\alpha \in(0, \pi)$, then it follows that

$$
\min _{i \in\{1,2\}}\left\{\frac{1}{1+2 x y \cos \alpha_{i}+(x y)^{2}}\right\}=\frac{1}{1+2 x y \cos \alpha+(x y)^{2}},
$$

and by Lemma 2.1, we can obtain

$$
\omega(x)=\frac{\pi}{\sin \alpha}(x \neq 0) .
$$


Lemma 2.2. If $p>1, \frac{1}{p}+\frac{1}{q}=1,0<\alpha_{1}<\alpha_{2}<\pi, f(x)$ is a non-negative measurable function in $(-\infty, \infty)$, then we have

$$
\begin{aligned}
J & :=\int_{-\infty}^{\infty}|y|^{p-1}\left(\int_{-\infty}^{\infty} \min _{i \in\{1,2\}}\left\{\frac{1}{1+2 x y \cos \alpha_{i}+(x y)^{2}}\right\} f(x) d x\right)^{p} d y \\
& \leq k^{p} \int_{-\infty}^{\infty}|x|^{-1} f^{p}(x) d x .
\end{aligned}
$$

Proof. By Lemma 2.1 and Hölder's inequality [13], we have

$$
\begin{aligned}
& \left(\int_{-\infty}^{\infty} \min _{i \in\{1,2\}}\left\{\frac{1}{1+2 x y \cos \alpha_{i}+(x y)^{2}}\right\} f(x) d x\right)^{p} \\
\leq & \int_{-\infty}^{\infty} \min _{i \in\{1,2\}}\left\{\frac{1}{1+2 x y \cos \alpha_{i}+(x y)^{2}}\right\} f^{p}(x) d x \\
& \times\left(\int_{-\infty}^{\infty} \min _{i \in\{1,2\}}\left\{\frac{1}{1+2 x y \cos \alpha_{i}+(x y)^{2}}\right\} d x\right)^{p-1} \\
= & k^{p-1}|y|^{-p+1} \int_{-\infty}^{\infty} \min _{i \in\{1,2\}}\left\{\frac{1}{1+2 x y \cos \alpha_{i}+(x y)^{2}}\right\} f^{p}(x) d x .
\end{aligned}
$$

Then by Fubini's theorem, it follows

$$
\begin{aligned}
J & \leq \int_{-\infty}^{\infty}\left[\int_{-\infty}^{\infty} \min _{i \in\{1,2\}}\left\{\frac{1}{1+2 x y \cos \alpha_{i}+(x y)^{2}}\right\} f^{p}(x) d x\right] d y \\
& =k^{p-1} \int_{-\infty}^{\infty} \omega(x)|x|^{-1} f^{p}(x) d x=k^{p} \int_{-\infty}^{\infty}|x|^{-1} f^{p}(x) d x .
\end{aligned}
$$

The lemma is proved.

\section{Main Results and Applications}

Theorem 3.1. If $p>1, \frac{1}{p}+\frac{1}{q}=1,0<\alpha_{1}<\alpha_{2}<\pi, f, g \geq 0$, satisfying $0<$ $\int_{-\infty}^{\infty}|x|^{-1} f^{p}(x) d x<\infty$ and $0<\int_{-\infty}^{\infty}|y|^{-1} g^{q}(y) d y<\infty$, then we have

$$
\begin{aligned}
I & :=\int_{-\infty}^{\infty} \int_{-\infty}^{\infty} \min _{i \in\{1,2\}}\left\{\frac{1}{1+2 x y \cos \alpha_{i}+(x y)^{2}}\right\} f(x) g(y) d x d y \\
& <k\left(\int_{-\infty}^{\infty}|x|^{-1} f^{p}(x) d x\right)^{\frac{1}{p}}\left(\int_{-\infty}^{\infty}|y|^{-1} g^{q}(y) d y\right)^{\frac{1}{q}}, \\
J & =\int_{-\infty}^{\infty}|y|^{p-1}\left(\int_{-\infty}^{\infty} \min _{i \in\{1,2\}}\left\{\frac{1}{1+2 x y \cos \alpha_{i}+(x y)^{2}}\right\} f(x) d x\right)^{p} d y \\
& <k^{p} \int_{-\infty}^{\infty}|x|^{-1} f^{p}(x) d x,
\end{aligned}
$$


where the constant factor $k$ and $k^{p}$ are the best possible and $k$ is defined by (2.3). Inequality (3.1) and (3.2) are equivalent.

Proof. First, we prove (3.2). If (2.4) takes the form of equality for a $y \in(-\infty, 0) \cup$ $(0, \infty)$, then there exists constants $A$ and $B$, such that they are not all zero, and $A f^{p}(x)=B g^{q}(y)$ a.e. in $(-\infty, 0) \cup(0, \infty)$. Hence, there exists a constant $C$, such that $A f^{p}(x)=B g^{q}(y)=C$ a.e. in $(0, \infty)$. We suppose $A \neq 0$ (otherwise $B=A=$ $0)$. Then $|x|^{-1} f^{p}(x)=\frac{C}{A|x|}$ a. e. in $(-\infty, \infty)$, which contradicts the fact that $0<\int_{-\infty}^{\infty}|x|^{-1} f^{p}(x) d x<\infty$. Hence (2.4) takes the form of strict inequality; so does (3.2), and we have (3.2).

Then we prove that they are equivalent. By Hölder's inequality [13], we have

$$
\begin{aligned}
I & =\int_{-\infty}^{\infty}\left(|y|^{\frac{1}{q}} \int_{-\infty}^{\infty} \min _{i \in\{1,2\}}\left\{\frac{1}{1+2 x y \cos \alpha_{i}+(x y)^{2}}\right\} f(x) d x\right)\left(|y|^{-\frac{1}{q}} g(y) d y\right) \\
& \leq J^{\frac{1}{p}}\left(\int_{-\infty}^{\infty}|y|^{-1} g^{q}(y) d y\right)^{\frac{1}{q}} .
\end{aligned}
$$

By (3.2), we have (3.1). On the other hand, suppose that (3.1) is valid. Setting

$$
g(y)=|y|^{p-1}\left(\int_{-\infty}^{\infty} \min _{i \in\{1,2\}}\left\{\frac{1}{1+2 x y \cos \alpha_{i}+(x y)^{2}}\right\} f(x) d x\right)^{p-1},
$$

then it follows $J=\int_{-\infty}^{\infty}|y|^{-1} g^{q}(y) d y$. By (2.3), we have $J<\infty$. If $J=0$, then (3.2) is obvious valid; if $0<J<\infty$, then by (3.1), we obtain

$$
\begin{gathered}
0<\int_{-\infty}^{\infty}|y|^{-1} g^{q}(y) d y=J=I \\
<k\left(\int_{-\infty}^{\infty}|x|^{-1} f^{p}(x) d x\right)^{\frac{1}{p}}\left(\int_{-\infty}^{\infty}|y|^{-1} g^{q}(y) d y\right)^{\frac{1}{q}}, \\
J^{\frac{1}{p}}=\left(\int_{-\infty}^{\infty}|y|^{-1} g^{q}(y) d y\right)^{\frac{1}{p}}<k\left(\int_{-\infty}^{\infty}|x|^{-1} f^{p}(x) d x\right)^{\frac{1}{p}} .
\end{gathered}
$$

Hence we have (3.2), which is equivalent to (3.1).

Finally, we prove the factors are best possible. For $\varepsilon>0$, define functions $\tilde{f}(x), \tilde{g}(x)$ as follows:

$$
\begin{gathered}
\tilde{f}(x):= \begin{cases}x^{-\frac{2 \varepsilon}{p}}, & x \in(1, \infty), \\
0, & x \in[-1,1], \\
(-x)^{-\frac{2 \varepsilon}{p}}, & x \in(-\infty,-1) ;\end{cases} \\
\tilde{g}(x):= \begin{cases}x^{\frac{2 \varepsilon}{q}}, & x \in(0,1), \\
0, & x \in(-\infty,-1) \cup[1, \infty), \\
(-x)^{\frac{2 \varepsilon}{q}}, & x \in(-1,0) .\end{cases}
\end{gathered}
$$


then $\tilde{L}:=\left\{\int_{-\infty}^{\infty}|x|^{-1} \tilde{f}^{p}(x) d x\right\}^{\frac{1}{p}}\left\{\int_{-\infty}^{\infty}|y|^{-1} \tilde{g}^{q}(y) d y\right\}^{\frac{1}{q}}=\frac{1}{\varepsilon}$ and

$$
\tilde{I}:=\int_{-\infty}^{\infty} \int_{-\infty}^{\infty} \min _{i \in\{1,2\}}\left\{\frac{1}{1+2 x y \cos \alpha_{i}+(x y)^{2}}\right\} \tilde{f}(x) \tilde{g}(y) d x d y=I_{1}+I_{2}+I_{3}+I_{4}
$$

where,

$$
\begin{aligned}
& I_{1}:=\int_{-\infty}^{-1}(-x)^{-\frac{2 \varepsilon}{p}}\left[\int_{-1}^{0} \frac{(-y)^{\frac{2 \varepsilon}{q}}}{1+2 x y \cos \alpha_{1}+(x y)^{2}} d y\right] d x \\
& I_{2}:=\int_{-\infty}^{-1}(-x)^{-\frac{2 \varepsilon}{p}}\left[\int_{0}^{1} \frac{y^{\frac{2 \varepsilon}{q}}}{1+2 x y \cos \alpha_{2}+(x y)^{2}} d y\right] d x \\
& I_{3}:=\int_{1}^{\infty} x^{-\frac{2 \varepsilon}{p}}\left[\int_{-1}^{0} \frac{(-y)^{\frac{2 \varepsilon}{q}}}{1+2 x y \cos \alpha_{2}+(x y)^{2}} d y\right] d x \\
& I_{4}:=\int_{1}^{\infty} x^{-\frac{2 \varepsilon}{p}}\left[\int_{0}^{1} \frac{y^{\frac{2 \varepsilon}{q}}}{1+2 x y \cos \alpha_{1}+(x y)^{2}} d y\right] d x,
\end{aligned}
$$

By Fubini's theorem ${ }^{[14]}$, we obtain

$$
\begin{aligned}
I_{1}=I_{4} & =\int_{1}^{\infty} x^{-1-2 \varepsilon}\left(\int_{0}^{x} \frac{u^{\frac{2 \varepsilon}{q}}}{u^{2}+2 u \cos \alpha_{1}+1} d u\right) d x \quad(u=x y) \\
& =\int_{1}^{\infty} x^{-1-2 \varepsilon}\left(\int_{0}^{1} \frac{u^{\frac{2 \varepsilon}{q}} d u}{u^{2}+2 u \cos \alpha_{1}+1}+\int_{1}^{x} \frac{u^{\frac{2 \varepsilon}{q}} d u}{u^{2}+2 u \cos \alpha_{1}+1}\right) d x \\
& =\frac{1}{2 \varepsilon} \int_{0}^{1} \frac{u^{\frac{2 \varepsilon}{q}} d u}{u^{2}+2 u \cos \alpha_{1}+1}+\int_{1}^{\infty}\left(\int_{u}^{\infty} x^{-1-2 \varepsilon} d x\right) \frac{u^{\frac{2 \varepsilon}{q}} d u}{u^{2}+2 u \cos \alpha_{1}+1} \\
& =\frac{1}{2 \varepsilon}\left(\int_{0}^{1} \frac{u^{\frac{2 \varepsilon}{q}}}{u^{2}+2 u \cos \alpha_{1}+1} d u+\int_{1}^{\infty} \frac{u^{-\frac{2 \varepsilon}{p}}}{u^{2}+2 u \cos \alpha_{1}+1} d u\right), \\
I_{2}=I_{3}= & \frac{1}{2 \varepsilon}\left(\int_{0}^{1} \frac{u^{\frac{2 \varepsilon}{q}}}{u^{2}-2 u \cos \alpha_{2}+1} d u+\int_{1}^{\infty} \frac{u^{-\frac{2 \varepsilon}{p}}}{u^{2}-2 u \cos \alpha_{2}+1} d u\right) \cdot(u=-x y)
\end{aligned}
$$

In view of the above results, if the constant factor $k$ in (3.1) is not the best possible, then exists a positive number $\mathrm{K}$ with $K<k$, such that

$$
\begin{aligned}
& \int_{0}^{1} \frac{u^{\frac{2 \varepsilon}{q}}}{u^{2}+2 u \cos \alpha_{1}+1} d u+\int_{1}^{\infty} \frac{u^{-\frac{2 \varepsilon}{p}}}{u^{2}+2 u \cos \alpha_{1}+1} d u \\
& \quad+\int_{0}^{1} \frac{u^{\frac{2 \varepsilon}{q}} d u}{u^{2}-2 u \cos \alpha_{2}+1}+\int_{1}^{\infty} \frac{u^{-\frac{2 \varepsilon}{p}} d u}{u^{2}-2 u \cos \alpha_{2}+1}=\varepsilon \tilde{I}<\varepsilon K \tilde{L}=K .
\end{aligned}
$$


By Fatou's lemma [14] and (3.6), we have

$$
\begin{aligned}
k= & \int_{0}^{\infty} \frac{1}{u^{2}+2 u \cos \alpha_{1}+1} d u+\int_{0}^{\infty} \frac{1}{u^{2}-2 u \cos \alpha_{2}+1} d u \\
= & \int_{0}^{1} \lim _{\varepsilon \rightarrow 0^{+}} \frac{u^{\frac{2 \varepsilon}{q}}}{u^{2}+2 u \cos \alpha_{1}+1} d u+\int_{1}^{\infty} \lim _{\varepsilon \rightarrow 0^{+}} \frac{u^{-\frac{2 \varepsilon}{p}}}{u^{2}+2 u \cos \alpha_{1}+1} d u \\
& +\int_{0}^{1} \lim _{\varepsilon \rightarrow 0^{+}} \frac{u^{\frac{2 \varepsilon}{q}}}{u^{2}-2 u \cos \alpha_{2}+1} d u+\int_{1}^{\infty} \lim _{\varepsilon \rightarrow 0^{+}} \frac{u^{-\frac{2 \varepsilon}{p}}}{u^{2}-2 u \cos \alpha_{2}+1} d u \\
\leq & \lim _{\varepsilon \rightarrow 0^{+}}\left[\int_{0}^{1} \frac{u^{\frac{2 \varepsilon}{q}}}{u^{2}+2 u \cos \alpha_{1}+1} d u+\int_{1}^{\infty} \frac{u^{-\frac{2 \varepsilon}{p}}}{u^{2}+2 u \cos \alpha_{1}+1} d u\right. \\
& \left.+\int_{0}^{1} \frac{u^{\frac{2 \varepsilon}{q}}}{u^{2}-2 u \cos \alpha_{2}+1} d u+\int_{1}^{\infty} \frac{u^{-\frac{2 \varepsilon}{p}}}{u^{2}-2 u \cos \alpha_{2}+1} d u\right] \leq K,
\end{aligned}
$$

which contradicts the fact that $K<k$. Hence the constant factor $k$ in (3.1) is the best possible.

If the constant factor in (3.2) is not the best possible, then by (3.3), we may get a contradiction that the constant factor in (3.1) is not the best possible. Thus the theorem is proved.

In view of Note (2) and Theorem 3.1, we still have

Theorem 3.2. If $p>1, \frac{1}{p}+\frac{1}{q}=1,0<\alpha<\pi, f, g \geq 0$, satisfying $0<$ $\int_{-\infty}^{\infty}|x|^{-1} f^{p}(x) d x<\infty$ and $0<\int_{-\infty}^{\infty}|y|^{-1} g^{q}(y) d y<\infty$, then we have

$$
\begin{aligned}
& \int_{-\infty}^{\infty} \int_{-\infty}^{\infty} \frac{1}{1+2 x y \cos \alpha+(x y)^{2}} f(x) g(y) d x d y \\
< & \frac{\pi}{\sin \alpha}\left(\int_{-\infty}^{\infty}|x|^{-1} f^{p}(x) d x\right)^{\frac{1}{p}}\left(\int_{-\infty}^{\infty}|y|^{-1} g^{q}(y) d y\right)^{\frac{1}{q}}, \\
& \int_{-\infty}^{\infty}|y|^{p-1}\left(\int_{-\infty}^{\infty} \frac{1}{1+2 x y \cos \alpha+(x y)^{2}} f(x) d x\right)^{p} d y \\
< & {\left[\frac{\pi}{\sin \alpha}\right]^{p} \int_{-\infty}^{\infty}|x|^{-1} f^{p}(x) d x, }
\end{aligned}
$$

where the constant factor $\frac{\pi}{\sin \alpha}$ and $\left[\frac{\pi}{\sin \alpha}\right]^{p}$ are the best possible. Inequality (3.8) and (3.9) are equivalent.

In particular, for $\alpha=\pi / 3$, we have the following equivalent inequalities:

$$
\begin{aligned}
& \int_{-\infty}^{\infty} \int_{-\infty}^{\infty} \frac{1}{1+x y+(x y)^{2}} f(x) g(y) d x d y \\
< & \frac{2 \pi}{\sqrt{3}}\left(\int_{-\infty}^{\infty}|x|^{-1} f^{p}(x) d x\right)^{\frac{1}{p}}\left(\int_{-\infty}^{\infty}|y|^{-1} g^{q}(y) d y\right)^{\frac{1}{q}},
\end{aligned}
$$




$$
\begin{aligned}
& \int_{-\infty}^{\infty}|y|^{p-1}\left(\int_{-\infty}^{\infty} \frac{1}{x^{2}+x y+y^{2}} f(x) d x\right)^{p} d y \\
< & {\left[\frac{2 \pi}{\sqrt{3}}\right]^{p} \int_{-\infty}^{\infty}|x|^{-1} f^{p}(x) d x . }
\end{aligned}
$$

Theorem 3.3. As the assumptions of Theorem 3.1, replacing $p>1$ by $0<p<1$, we have the equivalent reverses of (3.1) and (3.2) with the best constant factors.

Proof. By the reverse Hölder's inequality ${ }^{[13]}$, we have the reverse of (2.3) and (3.3). It is easy to obtain the reverse of (3.2). In view of the reverses of (3.2) and (3.3), we obtain the reverse of (3.1). On the other hand, suppose that the reverse of (3.1) is valid. Setting the same $g(y)$ as the proof of theorem 3.1, by the reverse of (2.3), we have $J>0$. If $J=\infty$, then the reverse of (3.2) is obvious valid; if $J<\infty$, then by the reverse of (3.1), we obtain the reverses of (3.4) and (3.5). Hence we have the reverse of (3.2), which is equivalent to the reverse of (3.1).

If the constant factor $k$ in the reverse of (3.1) is not the best possible, then there exists a positive constant $K$ (with $K>k$ ), such that the reverse of (3.1) is still valid as we replace $k$ with $K$. By the reverse of (3.6), we have

$$
\begin{aligned}
& \int_{0}^{1}\left[\frac{1}{u^{2}+2 u \cos \alpha_{1}+1}+\frac{1}{u^{2}-2 u \cos \alpha_{2}+1}\right] u^{\frac{2 \varepsilon}{q}} d u \\
& \quad+\int_{1}^{\infty}\left[\frac{1}{u^{2}+2 u \cos \alpha_{1}+1}+\frac{1}{u^{2}-2 u \cos \alpha_{2}+1}\right] u^{-\frac{2 \varepsilon}{p}} d u>K .
\end{aligned}
$$

For $\varepsilon \rightarrow 0^{+}$, by the Levi's theorem ${ }^{[14]}$, we find

$$
\begin{aligned}
& \int_{1}^{\infty}\left[\frac{1}{u^{2}+2 u \cos \alpha_{1}+1}+\frac{1}{u^{2}-2 u \cos \alpha_{2}+1}\right] u^{-\frac{2 \varepsilon}{p}} d u \\
& \quad \rightarrow \int_{1}^{\infty}\left[\frac{1}{u^{2}+2 u \cos \alpha_{1}+1}+\frac{1}{u^{2}-2 u \cos \alpha_{2}+1}\right] d u,
\end{aligned}
$$

For $0<\varepsilon<\varepsilon_{0}, q<0$, such that $1+\frac{2 \varepsilon_{0}}{q}>0$, since $u^{\frac{2 \varepsilon}{q}} \leq u^{\frac{2 \varepsilon_{0}}{q}}, u \in[0,1)$, and

$$
\begin{aligned}
& \int_{0}^{1}\left[\frac{1}{u^{2}+2 u \cos \alpha_{1}+1}+\frac{1}{u^{2}-2 u \cos \alpha_{2}+1}\right] u^{\frac{2 \varepsilon_{0}}{q}} d u \\
= & \int_{0}^{1}\left[\frac{1}{\left(u+\cos \alpha_{1}\right)^{2}+\left(\sin \alpha_{1}\right)^{2}}+\frac{1}{\left(u-\cos \alpha_{2}\right)^{2}+\left(\sin \alpha_{2}\right)^{2}}\right] u^{\frac{2 \varepsilon_{0}}{q}} d u \\
\leq & {\left[\frac{1}{\left(\sin \alpha_{1}\right)^{2}}+\frac{1}{\left(\sin \alpha_{2}\right)^{2}}\right] \int_{0}^{1} u^{\frac{2 \varepsilon_{0}}{q}} d u=\left[\frac{1}{\left(\sin \alpha_{1}\right)^{2}}+\frac{1}{\left(\sin \alpha_{2}\right)^{2}}\right]\left(1+\frac{2 \varepsilon_{0}}{q}\right)<\infty, }
\end{aligned}
$$

then by Lebesgue control convergence theorem ${ }^{[14]}$, for $\varepsilon \rightarrow 0^{+}$, we have

$$
\int_{0}^{1}\left[\frac{1}{u^{2}+2 u \cos \alpha_{1}+1}+\frac{1}{u^{2}-2 u \cos \alpha_{2}+1}\right] u^{\frac{2 \varepsilon}{q}} d u \rightarrow
$$




$$
\int_{0}^{1}\left[\frac{1}{u^{2}+2 u \cos \alpha_{1}+1}+\frac{1}{u^{2}-2 u \cos \alpha_{2}+1}\right] d u .
$$

By (3.12), (3.13) and (3.14), for $\varepsilon \rightarrow 0^{+}$, we have $k \geq K$, which contradicts the fact that $k<K$. Hence the constant factor $k$ in the reverse of (3.1) is the best possible.

If the constant factor in reverse of (3.2) is not the best possible, then by the reverse of (3.3), we may get a contradiction that the constant factor in the reverse of (3.1) is not the best possible. Thus the theorem is proved.

By the same way of Theorem 3.3, we still have

Theorem 3.4. As the assumptions of Theorem 3.2, replacing $p>1$ by $0<p<1$, we have the equivalent reverses of (3.8) and (3.9) with the best constant factors.

\section{References}

[1] G. H. Hardy, J. E. Littlewood, G. Polya, Inequalities, Cambridge University Press, Cambridge, UK, 1952.

[2] J. Kuang, Introudction to Real Analysis. Hunan Educiton Press, Changsha, China, 1996.

[3] J. Kuang, Applied inequalities, Shangdong Science and Technology Press, Jinan, China, 2004.

[4] D. S. Mitrinović, J. E. Pecaric, A. M. Fink, Inequalities involving functions and their integrals and deivatives, vol.53, Kluwer Academic, Boston, USA, 1991.

[5] Y. Ping, H. Wang and L. Song, Complex function, Science Press, Beijing, China, 2004.

[6] J. Xu, Hardy-Hilbert's inequalities with two parameters, Advances in Mathematics, 36(2)(2007), 63-76.

[7] D. Xin, A Hilbert-type integral inequality with the homogeneous kernel of zero degree, Mathematical Theory and Applications, 30(2)(2010), 70-74.

[8] B. Yang, On the norm of an integral operator and applications, J. Math. Anal. Appl., 321(2006), 182-192.

[9] B. Yang, A new Hilbert-type inequality, Bull. Belg. Math. Soc., 13(2006), 479-487.

[10] B. Yang, On the norm of a Hilbert's type linear operator and applications, J. Math. Anal. Appl., 325(2007), 529-541.

[11] B. Yang, A survey of the study of Hilbert-type inequalities with parameters, Advances in Mathematics, 38(3)(2009), 257-268.

[12] B. Yang, The norm of operator and Hilbert-type inequalities, Science Press, Beijing, China, 2009.

[13] B. Yang, A Hilbert-type integral inequality with the homogenous kernel of degree 0, Journal of Shandong University (Natural Science), 45(2)(2010), 103-106. 
[14] Z. Zeng, Z. Xie, On a new Hilbert-type integral inequality with the homogeneous kernel of degree 0 and the integral in whole plane, Journal of Inequalities and Applications, (2010), Article ID 256796, 9 pages. 